\title{
Evaluation of laser sensors for precise line following applications
}

\author{
Michael Albert ${ }^{1}$, Daniel Eck ${ }^{1}$, Klaus Schilling ${ }^{2}$ \\ ${ }^{1}$ Zentrum für Telematik e.V., Allesgrundweg 12, 97218 Gerbrunn, Germany, \\ m.albert@telematik-zentrum.de, d.eck@telematik-zentrum.de \\ ${ }^{2}$ Department of Computer Science - Robotics and Telematics, University of Wuerzburg, Germany \\ schi@informatik.uni-wuerzburg.de
}

\begin{abstract}
For the development of a line coating robot, that is able to autonomously apply line markings on the ground, the robot needs to follow a line with high precision. To minimize the costs of the system a low cost laser sensor was developed for navigating the system along a laser line. The sensor was then compared to a commercial laser receiver for rotating lasers. The system was tested in an application oriented scenario and tracked with a high precision positioning system to calculate the deviation of the system to the optimal path.
\end{abstract}

Key words: laser sensor, line follow, mobile robotics, powder coating

\section{Introduction}

In many areas ground markings are needed, for example to signal driveways for forklifts or escape routes. Such markings are mainly applied by hand with spray paint or adhesive tapes which have to be renewed every few month. We built a system that uses an adapted powder coating technique to create very durable markings on different surfaces. A robot system is used to navigate the system precisely with the help of laser sensors. Figure 1 visualizes the concept of the whole system. To reduce costs of the system we developed a cheap laser sensor to navigate the system along a laser line. We then compared our sensor to a commercial laser sensor with respect to robustness, accuracy and performance.

Figure 1: Concept of the line coating system

\section{State of the Art}

The idea of building a robot that follows a given line is nearly as old as mobile robotics itself [1][2]. In lessons about mobile robotics there is often an exercise that includes building a line following robot. These robots mostly use light and photo diodes to detect a color difference on the floor. With a simple hysteresis controller a line follow navigation for the robot can be implemented. The principle of controlling the robot with light and optical sensors can be extended to a laser point, that is guiding the robot, and a two dimensional optical sensor that tracks the laser point, like Hara [3] did. This is a good possibility to guide the robot but needs a laser pointer mounted at the ceiling that can be pointed in different directions. It is also not applicable outdoors.

Industrial production halls sometimes use automated transport systems that are usually

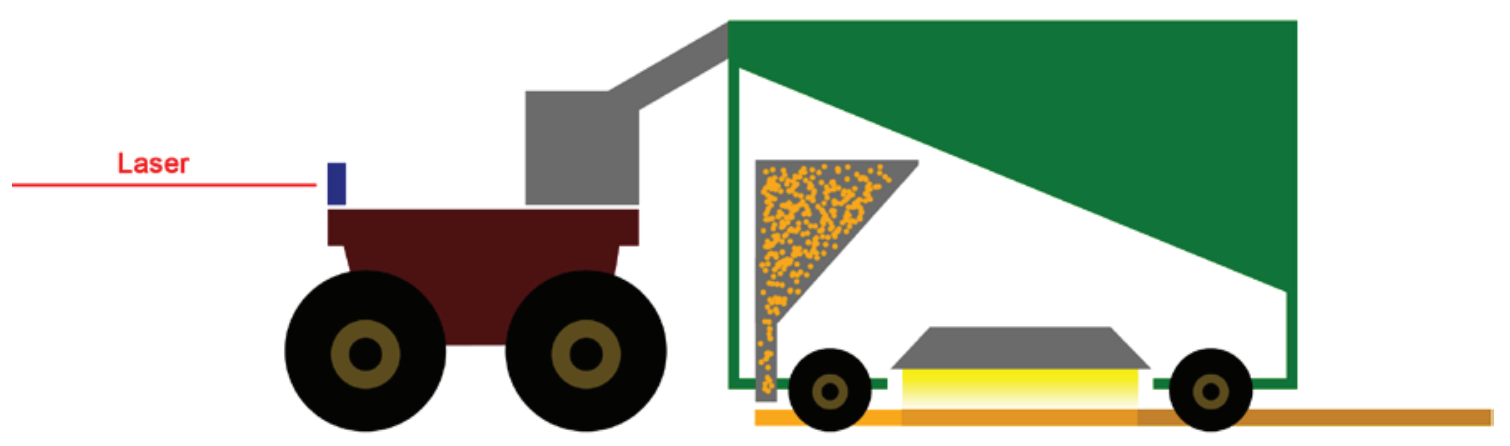


line following systems [4][5]. The line following in these systems is realized by magnetic spikes or tapes which are detected by magnetic sensors at the transport system. Alternatively, inductive loops can be used to guide the system along the intended path. Both techniques require permanent installations on the surface which is no option for a mobile system.

Navigating a system with external reference to drive straight lines is also a problem in agriculture. To maximize the productivity of the field the farmers want to plant the crop in exact rows. Usually the global positioning system (GPS) is used to raise efficiency on planting crop but lately the use of laser line guided systems became popular in agriculture [6].

\section{Development of a laser sensor}

We decided to use a simple commercial line laser to mark the line where our system should apply the coating on the surface. The laser line has two great advantages: the line can be seen by eye, so that the worker can plan and place the laser line exactly where the marking on the floor should be placed. The laser line can also be used to guide the line coating robot precisely along the path. As it has to be set up for planning and measuring the markings it is a 'built in' external reference for the robot.

The system has to be competitive with standard marking techniques, like painting or adhesive tapes, so it has to be as cheap as possible. We decided to use a standard line laser for home use like the Bosch GLL2 and develop our own low cost laser sensor.

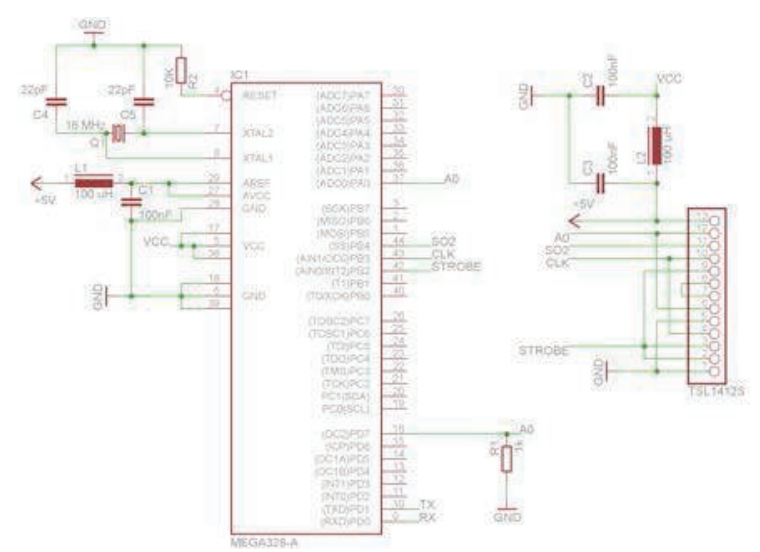

Figure 2: Schematic of our low cost laser sensor

Like in [7] we used the linear image sensor TSL1412s from TAOS and an Atmel microcontroller to read the brightness values from the sensor. The image sensor has a sensor area of 1536 pixels with a resolution of $400 \mathrm{dpi}$. The resolution is high enough to detect the laser line on several pixels and therefore calculate the position of the laser line on the sensor very precisely. Figure 2 shows the wiring diagram of the electronics used to read the sensor values and transmit them to the computer that controls the system.

The sensor is mounted on the front of the robot and is pointing directly to the laser emitter. Therefore, the position of the laser is the brightest spot on the sensor array.

When we analyzed the first values of the sensor we discovered that the sensor is very sensitive and overexposes in normal ambient light. We attached a neutral density filter in front of the sensor to lower the whole amount of light that reaches the sensor. In addition, we added two foils that are used for the lighting in theaters. The first is a red one called 'light red' that filters most of the light spectrum below our laser wavelength of about $635 \mathrm{~nm}$. The second foil, which is called 'thermashield', is originally used to deflect heat and is therefore great to deflect light with a wavelength higher than $700 \mathrm{~nm}$ as depicted in Figure 3.

After filtering the ambient light we could detect the laser spot on the sensor very well. A baseline correction on the sensor values allows the use of a dynamic trigger threshold.

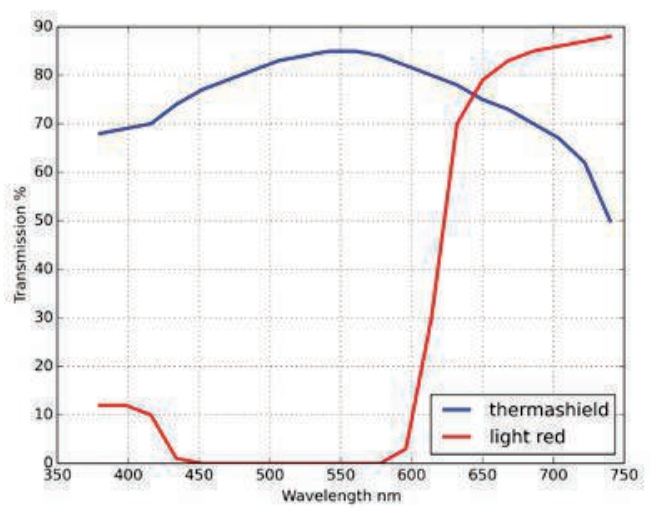

Figure 3: Light transmitted through the different filters

Figure 4 shows the values of the sensor where the lower horizontal line represents the baseline correction and the upper horizontal line is the dynamic trigger threshold. The peak is the laser on the sensor from a distance of about $15 \mathrm{~m}$. For convenience we assume that the exact center of the laser line is the center point between the points where the sensor values intersect the trigger threshold. These points are marked with small circles in the figure. 


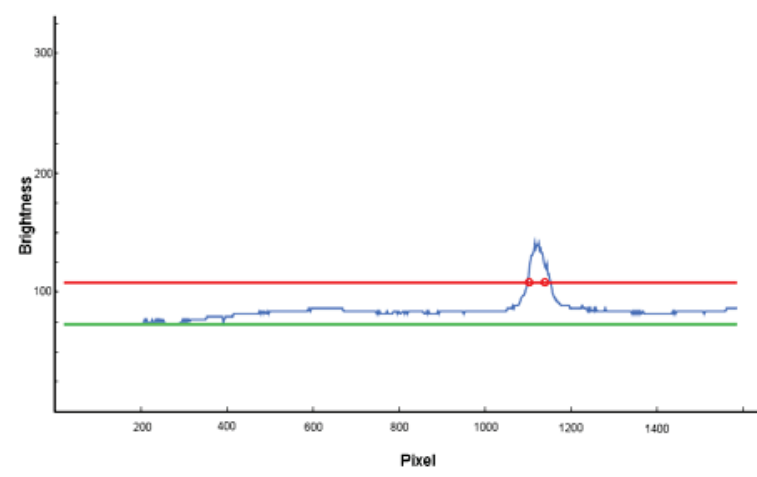

Figure 4: Indoor detection of the laser spot on the self-made laser sensor with baseline correction (lower line) and dynamic trigger threshold (upper line).

\section{Comparison of sensors}

To evaluate our sensor we build up an identical system with a commercial handheld laser sensor that was adapted to be machine readable. We used a laser receiver from $\mathrm{Nedo}^{1}$ that is used in combination with a rotating laser that is designed to be used on construction sites. While the commercial laser sensor uses a very high resolution inside we can only read 4 bit values from the sensor. The sensor has a switchable accuracy that defines how far the laser has to travel on the sensor area to change the output value. The accuracy can be switched between $4 \mathrm{~mm}, 2 \mathrm{~mm}, 1 \mathrm{~mm}$ and $0.5 \mathrm{~mm}$. During our tests we left the setting at $0.5 \mathrm{~mm}$ to get the highest accuracy.

To compare the two different systems we build a test scenario where the system followed a 10 meter straight laser line. We tracked the robots position with the iSpace system ${ }^{2}$ to calculate the difference from the optimal path which is represented by the laser line and was also measured with the tracking system to get the reference into the same system. The iSpace system, produced by Nikon Metrology, is an optical laser based metrology and tracking system suitable for indoor and outdoor applications. Rotating transmitters emit laser beams, which are detected by sensor frames. These sensor frames consists of at least one photo sensor, connected to a communication module (PCE). The PCE forwards the sensor data to the iSpace control software, which is running on a dedicated PC. Finally, the iSpace control software calculates and saves the position information of the sensor frame. Typically, the accuracy of the system is less than $0.25 \mathrm{~mm}$ with a sampling rate of $40 \mathrm{~Hz}$ [8].

\footnotetext{
${ }^{1} \mathrm{http}: / /$ www.nedo.com/

2 http://www.metris3d.hu/termekeink/

nagy_volumenu_cmm_merogepek/ispace.html
}

The robot is driving with an average speed of $18 \mathrm{~mm}$ per second. The slow speed is a requirement of the curing process which forces us to drive at about 1 meter per minute.

We ran several tests with both sensors. Table 1 shows some results of two test runs for each sensor. We measured the mean, variance, standard deviation and maximum of the distance between the driven path of the robot and the laser line. The results of the sensors show a similar precision. For our use case the goal is to draw an 'optically straight line' which is very hard to quantify. The system will draw lines with a thickness of about $5 \mathrm{~cm}$ where deviations of a few millimeters won't matter.

The test results were produced with our first prototype of the system which uses a simple hysteresis controller to correct the orientation of the robot. We have implemented an advanced version of the controller which is still untested but we expect even better results.

Table 1: Results for the $10 \mathrm{~m}$ driving test

\begin{tabular}{|c|c|c|c|c|}
\hline \multirow{2}{*}{$\begin{array}{l}\text { all } \\
\text { values } \\
\text { in } \mathrm{mm}\end{array}$} & \multicolumn{2}{|c|}{$\begin{array}{l}\text { low cost laser } \\
\text { sensor }\end{array}$} & \multicolumn{2}{|c|}{$\begin{array}{ll}\text { commercial laser } \\
\text { receiver }\end{array}$} \\
\hline & $\begin{array}{l}\text { whole } \\
\text { run }\end{array}$ & $\begin{array}{l}\text { after } 1 \mathrm{~m} \\
\text { driving }\end{array}$ & $\begin{array}{l}\text { whole } \\
\text { run }\end{array}$ & $\begin{array}{l}\text { after } 1 \mathrm{~m} \\
\text { driving }\end{array}$ \\
\hline mean & 0.6157 & 0.5927 & 2.7247 & 2.7550 \\
\hline std & 0.4128 & 0.4055 & 1.5631 & 1.7587 \\
\hline nax & 1.8266 & 1.7637 & 7.0807 & 6.8248 \\
\hline rms & 0.7413 & 0.7181 & 3.1412 & 3.2685 \\
\hline
\end{tabular}

For each test run we measured continuously the difference between the real position of the robot and the optimal path which is represented by the laser line. It is measured as the orthogonal distance from the measured real position of the robot to the ideal line. We compared the test runs by several values that were computed of the error values: mean value, standard deviation, maximum value and root mean square. Table 1 shows the values for one test run for each of the sensors. We realized that the robot with the commercial sensor attached very often has to correct his initial position as the user doesn't position the robot correctly on the line. We assume that in the real application the robot will drive a short distance before he starts applying the line on the floor. Therefore, we recalculated the values after one meter of driving. The first column shows the values for the whole 10 meters of driving while the second column shows the results when the first meter is not considered. The results show no big difference after the first bit of driving. 
For a better understanding of the oscillation of the robot we plotted the error distribution of both sensors (Figures 5 and 6). One can see that the oscillation is rather stable over the time. That refutes our assumption that the oscillation is higher at the beginning of the run due to the varying positioning of the robot by the user.

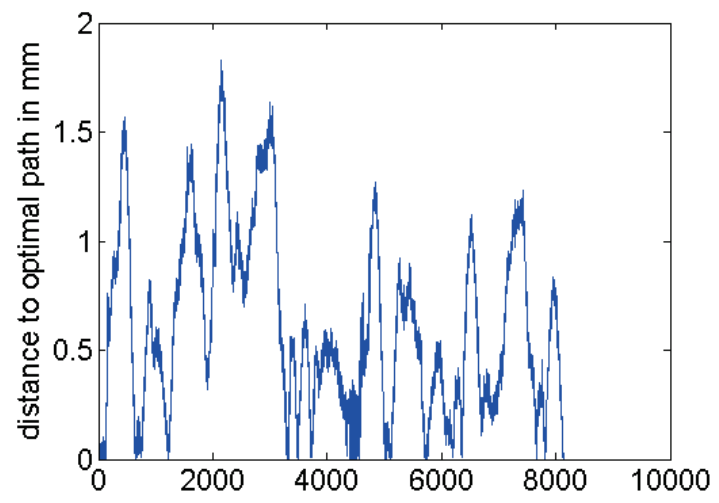

Figure 5: Error distribution - low cost laser sensor

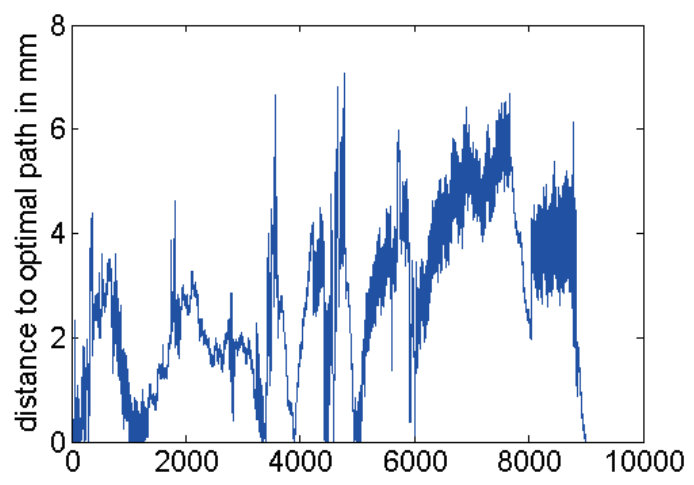

Figure 6: Error distribution - commercial laser receiver

The histogram of the error values for both sensors, depicted in figures 7 and 8 , form a good criteria for the quality of the driven line. Most of the errors are in the left area which means that they are rather small errors. The error values created by the commercial sensor are a bit higher in the maximum values and are more evenly distributed which leads to a lower quality of the driven line.

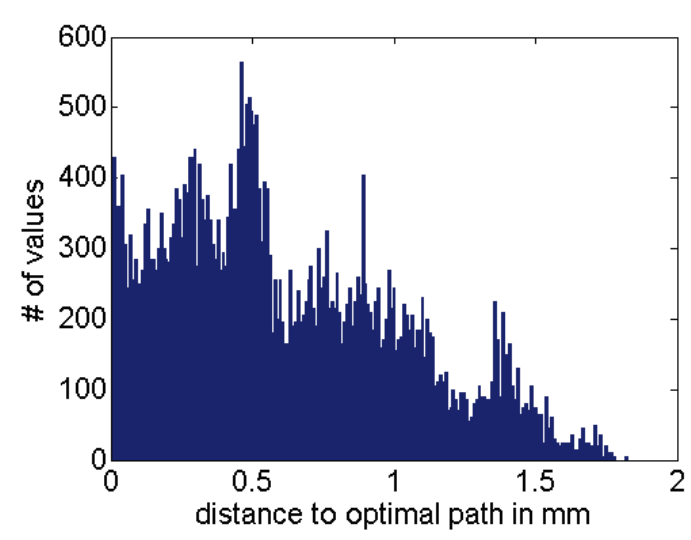

Figure 7: Histogram of the error values - low cost laser sensor

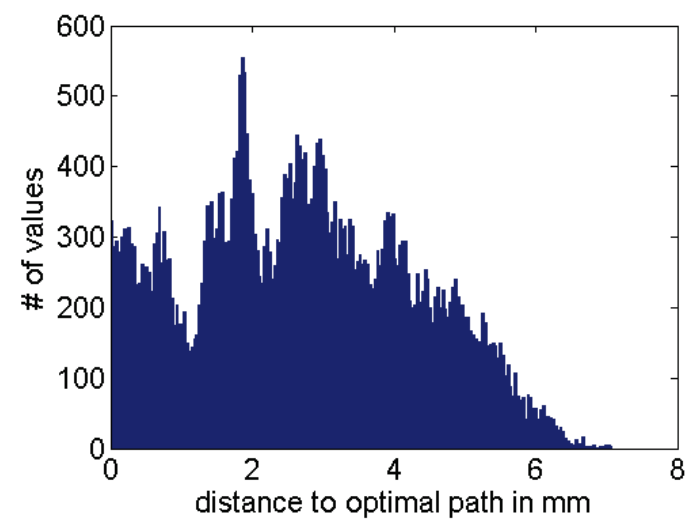

Figure 8: Histogram of the error values - commercial laser receiver

We tried to do some tests outside as well but in bright sunlight the image of our light sensor overexposes so that the laser cannot be detected reliable anymore. We tried to compensate the overexposure by adding filters but got similar or even worse results.

\section{Conclusion}

Our tests showed that the system reaches a similar accuracy with both of the sensors. Our self-made sensor allows a very high precision navigation but has great issues with bright ambient light. The commercial sensor uses the frequency of the rotating laser to lock on the signal and ignore the ambient light. It was surprising that our sensor gave better results than the commercial one. We assume that the higher sensitivity of the commercial sensor in combination with the simple hysteresis controller causes a higher oscillation effect. We have to run further tests to confirm this behavior.

All in all our low cost laser sensor is a very good alternative to the commercial version with the limitation that it is usable indoors only but comes at a price 10 times lower than the commercial one. 


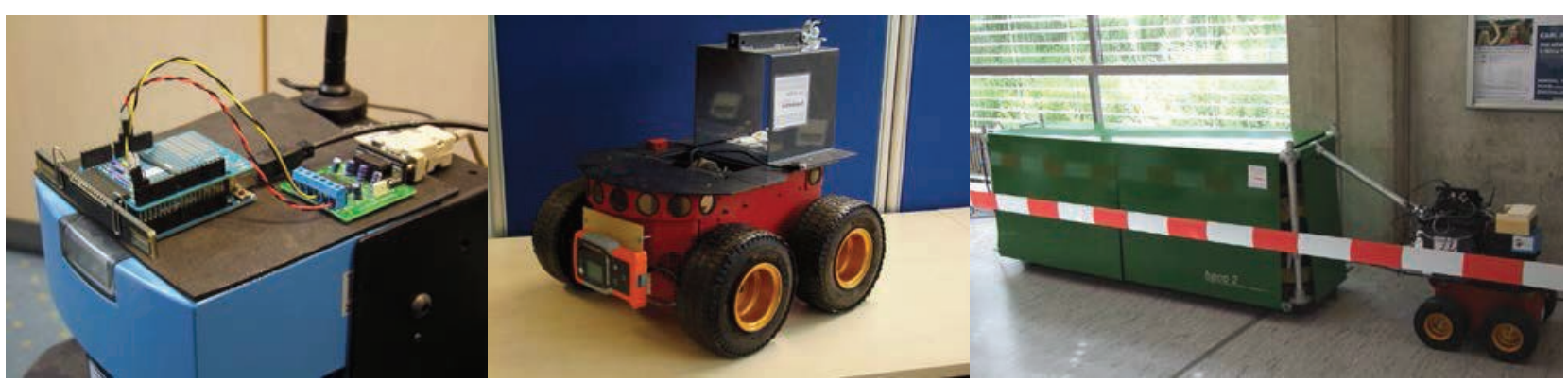

Figure 9: a) Prototype of the low cost laser sensor, b) Robot with attached commercial laser receiver, c) Prototype of the line coating robot

\section{Application}

The line follow technology was developed in a research project which aims to create a robot system that is capable of autonomously applying lines on different types of surfaces like roads, factory floors or the ground of swimming pools ${ }^{3}$.

Since markings on factory floors are usually made of tape or wet paint they are not very durable and have to be renewed every few month. Therefore, the line coating robot uses an adapted powder coating technique to apply markings on the ground. The powder coating technique promises high durable markings but is currently not available for markings on the ground. Powder coating is usually done in specific environments where the powder is applied with a spray gun which applies an electrostatic charge to the powder. The powder is then attracted to the grounded workpiece and forms an even surface. After application of the powder the part is put in a curing oven to complete the powder coating process.

In order to develop a system that can apply high durable markings on a surface we combined an adapted powder coating technique with a high precision navigating robot. The application of the powder was adapted to be available on a mobile and moving system and can be cured on site.

The line coating robot consists of two modules: The robot integrates the sensor with the driving platform and does computations and navigation to drive the system with high precision along the given path. It also commands the second module. The application unit applies the powder on the ground and controls the curing process. It is pulled by the robot and receives commands to start or stop powder application or the curing process. Figure 9 shows the prototypes for the two different sensors and the complete prototype consisting of the robot and the application unit.

\footnotetext{
${ }^{3}$ http://www.telematik-zentrum.de/projekte/ detailbeschreibung/details/libero.html
}

The first scenario is to place markings on the ground of swimming pools. These markings are needed to separate swimming lanes or line markings for water polo. The markings are usually painted with wet paint in case the pool is covered with tiles. The newer pools, which are lined with stainless steel, are sometimes already marked with powder coating technique. The bottom of the pool is then covered with premarked steel sheets that are composed to form the correct image. The markings are then intermittent by the welding seams. Our mobile powder coating system avoids the transport of all metal sheets and generates a seamless marking on the ground of such pools.

In factory halls markings are used to mark the path for fork-lifters and pedestrians or the required floor space of machines or parts. These markings are mostly straight lines, perpendicular to each other. Our test scenarios include long lines on concrete floors like those used for fork-lifters and rectangular markings like those for palettes.

The whole system is highly automated to be easily controlled and supervised by workers. Our prototype uses a game controller for intuitive driving of the robot and switching between the different states of the system.

\section{Future work}

We built a robust system that is easy to use and can autonomously create line markings on different surfaces. We built a low cost laser sensor that worked very well indoors, but suffers from overexposure in bright sunlight. Our low cost sensor was compared to a commercial one with both sensors yielding similar results. The accuracy of this laser line guided system was high enough to create straight lines with a high precision as claimed.

We are planning to refine the system in a few areas to extend the functionality of the system from straight lines to more complex forms. A first goal is to be able to mark arcs with different angles. The final step is to allow freeform markings. The application unit has to become smaller and can be improved to be more 
flexible. We already developed some concepts for improving the powder application and the curing process. We also did some tests and simulations on the quality of the navigation controller of the robot. We used our tracking system to provide an external position update to the robot and tested driving more complex forms. The results look promising and have to be tested on the real robot soon. The user interface has to be redesigned to allow a proper control of the system regarding complex forms. Currently it is planned to create an interface for smartphones or tablets to control the system. This provides much more options for feedback to the user and possibilities for advanced control techniques to drive the robot and control the whole system.

\section{Acknowledgement}

The authors gratefully acknowledge the personnel at Bader Pulverbeschichtung $\mathrm{GmbH}$ for the contributions to the project. In particular we acknowledge Matthias Bader who had the vision for such a system and supports a great cooperation in this research project.

The project in which context the described system is developed is supported by the Federal Ministry for Economic Affairs and Energy on the basis of a decision by the German Bundestag.

\section{References}

[1] Unyelioglu, K.A. and Hatipoglu, C. and Ozguner, U., Design and stability analysis of a lane following controller, IEEE Control Systems Technology Journal, 1,1997

[2] Faruk Bin Poyen, Santu Guin, Dibyendu Banerjee and Deep Mukherjee, Secured Robotic Line Follower Vehicle, Proceedings of the Intl. Conf. on Innovative Trends in Electronics Communication and Applications ICIECA2013, 2013

[3] Keigo Hara, Shoichi Maeyama and Akio Gofuku, Navigation Using a Laser for a Mobile Robot with a Optical Sensor Array, International Journal of Automation Technology, Sep. 2008

[4] D.M.K. Bong, Automatic Guided Vehicle System, Department of Electrical Engineering, University Tenega Nasional, Malaysia, 2004

[5] M.Mehdi Samaatiyan and Mehran Pakdaman, Design and implementation of line follower robot, Second International Conference on Computers and Electrical Engineering, 2009

[6] Yeyin Shi, Ning Wang, Randal K. Taylor, William R. Raun and James A. Hardin, Automatic corn plant location and spacing measurement using laser line-scan technique, Precision Agriculture, Oct. 2013
[7] I. Ciascai, L. Ciascai, Acquire images with a sensor and a microcontroller, EDN Network, September 2010

[8] L. Lemmer, R. Hess, M. Kraus, K. Schilling, Calibration of a Car-Like Mobile Robot with a High-Precision Positioning System, 2nd IFAC Symposium on Telematics Applications (2010), doi: 10.3182/20101005-4-RO-2018.00052

Supported by:

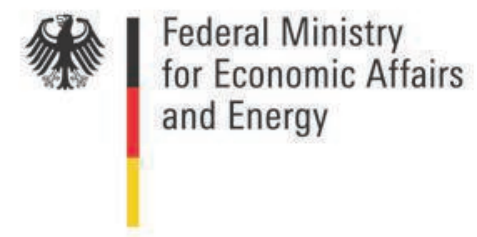

on the basis of a decision by the German Bundestag 